$$
\text { CONF-95/155-107 }
$$

Note: This is a preprint of paper being submitted for publication. Contents of this paper should not be quoted nor referred to without permission ot the author(s).

Invited paper, Symposium B, "Advanced Laser Processing of

Materials-Fundamentals and Applications, " 1995 Fall Meeting

of the Materials Research Society

\title{
Pulsed Laser Ablation Growth and Doping of Epitaxial Compound Semiconductor Films
}

Douglas H. Lowndes, Christopher M. Rouleau, D. B. Geohegan, A. A. Puretzky, ${ }^{*}$ M. A. Strauss, ${ }^{\ddagger}$ A. J. Pedraza, ${ }^{\ddagger}$ J. W. Park ${ }^{\ddagger}$, J. D. Budai, and D. B. Poker

$$
\begin{aligned}
& \text { "The submitted manuscript has been authored } \\
& \text { by a contractor of the U.S. Government under } \\
& \text { contract No. DE-ACOS-840R } 21400 \text {. } \\
& \text { Accordingly, the U.S. Government retains a } \\
& \text { nonexclusive, royalty-free license to publish or } \\
& \text { reproduce the published form of this } \\
& \text { contribution, or allow others to do so, for U.S. } \\
& \text { Government purposes." }
\end{aligned}
$$

\section{December 1995}

\footnotetext{
*Institute of Spectroscopy, Troitsk, Russia

$\ddagger$ Dept. of MS\&E, The University of Tennessee

Knoxville, Tennessee
}

Prepared by

Solid State Division

Oak Ridge National Laboratory

P.O. Box 2008

Oak Ridge, Tennessee 37831-6056

managed by

LOCKHEED MARTIN ENERGY SYSTEMS, INC

for the

U.S. DEPARTMENT OF ENERGY

under contract DE-AC05-84OR21400 


\section{DISCLAIMER}

This report was prepared as an account of work sponsored by an agency of the United States Goverament. Neither the United States Government nor any agency thereof, nor any of their employees, makes any warranty, express or implied, or assumes any legal liability or responsibility for the accuracy, completeness, or usefulness of any information, apparatus, product, or process disclosed, or represents that its use would not infringe privately owned rights. Reference herein to any specific commercial product, process, or service by trade name, trademark, manufacturer, or otherwise does not necessarily constitute or imply its endorsement, recommendation, or favoring by the United States Government or any agency thereof. The views and opinions of authors expressed herein do not necessarily state or reflect those of the United States Government or any agency thereof. 


\title{
PULSED LASER ABLATION GROWTH AND DOPING \\ OF EPITAXIAL COMPOUND SEMICONDUCTOR FILMS
}

\author{
DOUGLAS H. LOWNDES, ${ }^{*}$ CHRISTOPHER M. ROULEAU, ${ }^{*}$ D. B. GEOHEGAN, ${ }^{*}$ \\ A. A. PURETZKY, ${ }^{* *}$ M. A. STRAUSS, ${ }^{\dagger}$ A. J. PEDRAZA, ${ }^{\dagger}$ J. W. PARK ${ }^{\dagger}$, J. D. BUDAI, \\ AND D. B. POKER* \\ * Solid State Division, Oak Ridge National Laboratory, Oak Ridge, TN 37831-6056 \\ ** Institute of Spectroscopy, Troitsk, Russia \\ $\dagger$ Dept. of MS\&E, The University of Tennessee, Knoxville, TN 37996-2200
}

\begin{abstract}
Pulsed laser ablation (PLA) has several characteristics that are potentially attractive for the growth and doping of chemically complex compound semiconductors including (1) stoichiometric (congruent) transfer of composition from target to film, (2) the use of reactive gases to control film composition and/or doping via energetic-beam-induced reactions, and (3) low-temperature nonequilibrium phase formation in the laser-generated plasma "plume." However, the electrical properties of compound semiconductors are far more sensitive to low concentrations of defects than are the oxide metals/ceramics for which PLA has been so successful. Only recently have doped epitaxial compound semiconductor films been grown by PLA. Fundamental studies are being carried out to relate film electrical and microstructural properties to the energy distribution of ablated species, to the temporal evolution of the ablation pulse in ambient gases, and to beamassisted surface and/or gas-phase reactions. In this paper we describe results of ex situ Hall effect, high-resolution $\mathrm{x}$-ray diffraction, transmission electron microscopy, and Rutherford backscattering measurements that are being used in combination with in situ RHEED and time-resolved ion probe measurements to evaluate PLA for growth of doped epitaxial compound semiconductor films and heterostructures. Examples are presented and results analyzed for doped II-VI, I-III-VI, and column-III nitride materials grown recently in this and other laboratories.
\end{abstract}

\section{INTRODUCTION}

The past nine years have seen rapid growth in fundamental studies of the pulsed laser ablation process and in its application for pulsed laser deposition (PLD) of thin films. ${ }^{1}$ However, there has been relatively little use of PLD to grow doped, epitaxial compound (or elemental) semiconductor films. One reason for this is that current semiconductor technology is based on a relatively small set of materials for which highly specialized, successful, and in some cases high-throughput growth methods already have been developed. A second factor is that early PLD semiconductor films generally contained high areal densities of particulates and were polycrystalline and undoped, making them unsuitable for many applications. ${ }^{2}$ Contemporary investigators also may have been dissuaded by realization that the requirement to maintain stoichiometry is much more stringent for electronic semiconductors than for oxide ceramics (for example) since defect concentrations of only 1 part in $10^{-4}$ can prevent electrical activation of dopants. Furthermore, the energetic ablation beam may produce lattice displacement damage in semiconductor films during growth, resulting in point defects or defect complexes that could compensate deliberately introduced dopants.

Nevertheless, it is clear that PLA has potentially unique advantages for exploratory semiconductor materials research, especially for epitaxial growth of complex multi-element semiconductor materials and their inclusion in epitaxial superlattice or quantum well heterostructures, as follows.

(1) The capability to stoichiometrically (congruently) transfer material from a polycrystalline target to an epitaxial film is potentially important for growth of such underutilized semiconductor materials as the direct band gap ternary and multinary I-III-VI chalcopyrites (prototype: $\mathrm{CuInSe}_{2}$ ), which in epilayer form could provide a rich variety of energy band 
(2) The kinetic and internal energies of species in the ablation beam may be useful to reduce epitaxial film growth temperatures by enhancing surface diffusion.

(3) PLA's ability to deposit films in reactive (low-pressure ambient gas) environments is intriguing for growth of epitaxial column-III nitrides and their alloys for optoelectronic applications. ${ }^{4,5}$ The energetic ablation beam also can induce reactions with adsorbed or ambient gases such as $\mathrm{H}_{2} \mathrm{~S}$ and $\mathrm{N}_{2}$, to produce films whose composition ${ }^{6}$ and/or doping and electrical properties ${ }^{7,8}$ can be controlled by the ambient gas partial pressure.

(4) Dopants also can be incorporated directly in the ablation target and transferred to a film.9,10

(5) Pulsed deposition provides inherently "digital" precision in controlling film thickness, and can be carried out so that significantly sub-Ångstrom amounts of material are deposited with each laser pulse, yet attractive film-growth rates can be maintained at laser repetition rates of $\sim 10-40 \mathrm{~Hz}$.

Combining these capabilities with the use of a multi-target carousel in principle makes possible the growth of epitaxial quantum well or superlattice structures. Multinary targets could be used to precisely match lattice constants or band edge offsets in adjacent epilayers and to increase the range of band gaps and lattice constants available. Thus, PLA may make possible the growth of multinary-based epitaxial structures with compositional and doping profiles that are not easily achieved by conventional growth methods 6,11 for which the maintenance and controlled variation of multiple independent elemental fluxes may be difficult.

Toward this goal, several groups recently carried out experiments to determine whether PLA can be used to grow structurally high quality and highly doped epitaxial compound semiconductor films. ${ }^{4,5,7-10}$ We initiated a systematic study by focusing on the closely related II-VI and I-III-VI families of materials. The II-VI semiconductors with moderate to wide energy band gaps are interesting because the achievement of bipolar doping in them has been a formidable barrier using conventional growth methods. Hole (p-type) doping, in particular, has required highly reactive atomic species generated in non-equilibrium $\mathrm{RF}^{12}$ or DC 13,14 plasmas. Doping of II-VI films is important for optically active semiconductor devices ranging from diodes and lasers for displays and communications to photovoltaic energy conversion. Thus, the II-VIs provide a challenging binary and ternary materials field within which to evaluate PLD. The more complex I-III-VI chalcopyrite (ternary through pentenary) semiconductors are still relatively new and unexplored with regard to epitaxial growth. One of these materials, $\mathrm{CuIn}_{1-x} \mathrm{Ga}_{\mathbf{x}} \mathrm{Se}_{2}$, is the absorber in photovoltaic cells that currently hold the "world record" for solar energy conversion efficiency by a polycrystalline thin-film device. 15,16 However, the presence of grain boundaries, together with other complexities of polycrystalline film processing, have prevented obtaining a fundamental understanding of doping, $\mathrm{p}-\mathrm{n}$ junction formation, and intrinsic properties from photovoltaic materials. PLA's capacity for stoichiometric deposition may offer a significant advantage for such studies in epitaxial films, in comparison with other growth methods.

In this article we review recent progress using PLA to grow doped, epitaxial compound semiconductor films, with particular attention to crystalline quality and the achievement of high doping levels in the II-VI, I-III-VI, and column-III nitride families.

\section{KEY MATERIALS ISSUES}

In considering how best to grow doped epitaxial compound semiconductor films by PLA a number of questions must be answered. These include:

(1) Can PLA maintain stoichiometry to the required level of 1 part in $10^{4}$ or better?

(2) How should multinary, polycrystalline ablation targets be prepared? Powders (if used) must be of very high purity to avoid accidental compensation (or doping) and must be well-mixed. Low-density, cold-pressed targets are easy to make but produce high areal densities of particulates on films. Hot-pressing to obtain high density targets complicates the purity problem. 
(3) Does the energetic ablation beam cause lattice displacement damage? If so, can it be controlled?

(4) Are ambient gases advantageous? Collisions with ambient gas molecules can be used to "tune" the ablation beam's mean kinetic energy down into the range of a few $\mathrm{eV}$, below the threshold for lattice displacement damage and point defect generation. This energy range also is generally considered ideal for beam-assisted chemical reactions in which an adsorbed or ambient gas is used to modify film composition and/or doping. But what is the effect on epitaxial film quality of having gas atoms present on the growing film surface?

(5) How best to dope PLA epitaxial semiconductor films? Dopants can be incorporated in the ablation target, but the relatively low concentrations needed for even heavily doped semiconductors $\left(\sim 1\right.$ part in $10^{3}$ to $\left.10^{4}\right)$ complicate target synthesis and compositional homogeneity requirements. A low-pressure ambient gas is an alternative, but only for a limited number of dopants. Highly reactive atomic beams can be used to supplement the ablation flux, but only in high vacuum. Considerations of efficiency, convenience, and film quality all are involved.

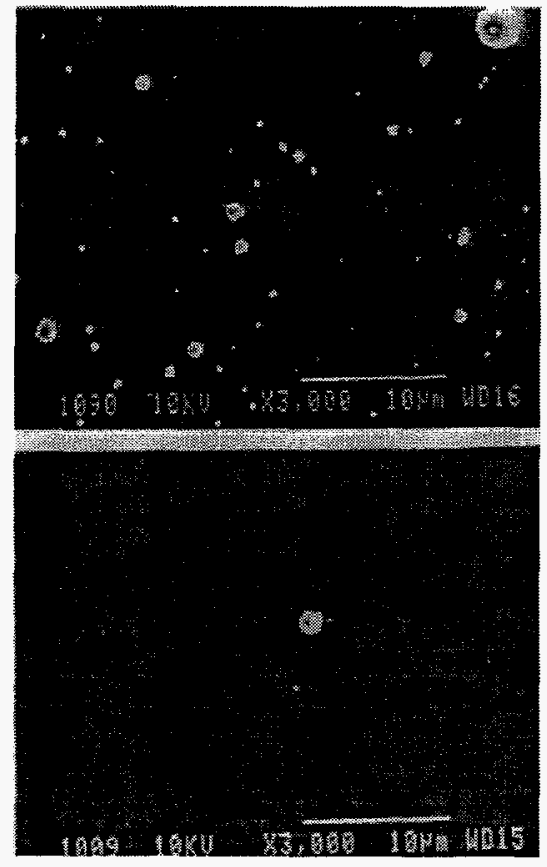

Figure 1. Particulates on films deposited at $\mathrm{D}_{\mathrm{ts}}=10 \mathrm{~cm}$ using (top) a cold-pressed $\mathrm{Zn}_{0.99} \mathrm{Mn}_{0.01}$ Te target $\left(\mathrm{E}_{\mathrm{d}}=2.6 \mathrm{~J} / \mathrm{cm}^{2}\right)$ and (bottom) a hot isostatically pressed $\mathrm{ZnTe}$ target $\left(\mathrm{E}_{\mathrm{d}}=1.9 \mathrm{~J} / \mathrm{cm}^{2}\right)$. Many comparable areas of the latter film were free of particulates.

\section{DOPED EPITAXIAL II-VI FILMS}

\section{Target Preparation}

The principal requirements in fabricating ablation targets for compound semiconductor growth are high purity and homogeneity and a high target density/thermal conductivity. High purity starting materials are essential so that PLA films are not accidentally doped, or deliberately introduced dopants unintentionally compensated. The use of high purity powders appears unavoidable to obtain semiconductor alloy compositions needed to tune energy band gaps and lattice constants. In the work described here, targets have been fabricated both by commercial hot pressing and by cold pressing finely ground and well mixed powders. The principal difference in the resulting films is a much higher areal density of $\sim \mu \mathrm{m}$-sized particulates on films grown from cold-pressed targets, as illustrated in Fig. 1. Most of the particulates in this case are irregular in shape with sharp features, indicating that they were produced when loosely bonded material and/or the finest powder grains simply fragmented off the target during ablation. In contrast, far fewer particulates are produced from hot-pressed targets and they are almost exclusively smaller (sub- $\mu \mathrm{m}$ ), round solidified droplets. These are believed to be caused by superheating and subsurface boiling that causes "spitting" or "splashing" of liquid droplets from the target. 17 However, the relatively high thermal conductivity of many semiconductor materials helps to minimize splashing. Consequently, the areal density of these droplets often can be reduced to a nearly negligible level $(0.01 \%<$ areal coverage $<0.1 \%$, see Fig. 1) in compound semiconductor films by using a high- density hot-pressed target.

\section{Stoichiometry}

Figure 2 shows results of Rutherford backscattering spectrometry (RBS) measurements of the $\mathrm{Zn} / \mathrm{Te}$ ratio in $-104-\mathrm{nm}$ thick $\mathrm{ZnTe}$ films that were deposited on Si substrates in vacuum and in 100 
mtorr $\mathrm{N}_{2}$ at $\mathrm{E}_{\mathrm{d}} \sim 0.66 \mathrm{~J} / \mathrm{cm}^{2} .{ }^{8}$ A Si substrate was used for RBS measurements, rather than GaAs, to avoid interferences between the $\mathrm{Zn}, \mathrm{Ga}$, and As RBS peaks. The horizontal lines at $1.00 \pm$ 0.015 represent the bounds of expected results for a series of RBS measurements on a $\mathrm{ZnTe}$ film with the ideal 1:1 stoichiometry. The ZnTe film deposited in 100 mtorr $\mathrm{N}_{2}$ is stoichiometric over much of its length, while the film deposited in vacuum is $\mathrm{Zn}$-deficient by a statistically significant amount near the center of the (rotating) substrate heater face. It is interesting that the PLA ZnTe film deposited in 100 mtorr $\mathrm{N}_{2}$ is stoichiometric within the accuracy of RBS because recent MBE growth of $\mathrm{ZnTe}$ required $\mathrm{Te} / \mathrm{Zn}$ flux ratios of 4 to 8 to achieve the

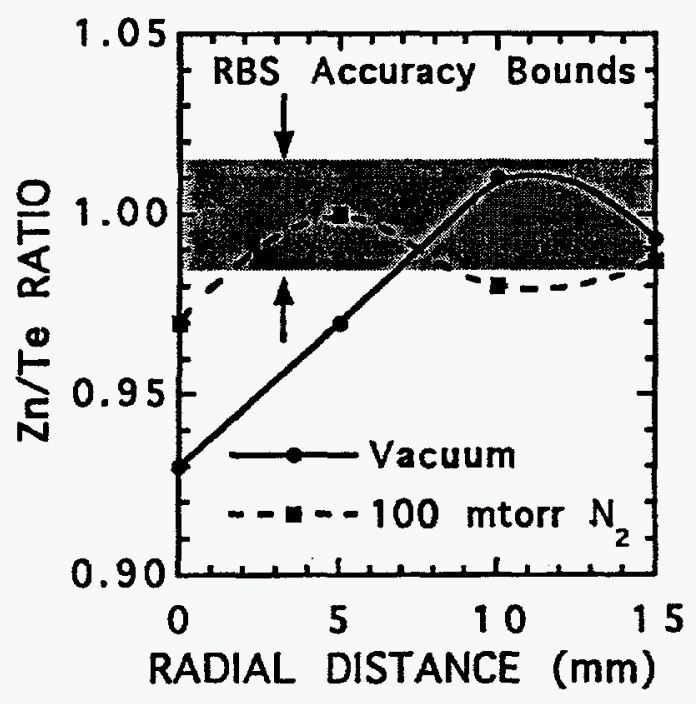

Figure 2. RBS measurements of the $\mathrm{Zn} / \mathrm{Te}$ ratio in $\mathrm{ZnTe}$ films deposited in vacuum $\left(<10^{-6}\right.$ Torr) and in 100 mtorr $\mathrm{N}_{2}$ (at $4 \mathrm{sccm}$ ) on a $\mathrm{Si}(001)$ substrate at $\mathrm{E}_{\mathrm{d}}=0.66 \mathrm{~J} / \mathrm{cm}^{2}$ and $\mathrm{T}=$ $320^{\circ} \mathrm{C}$. $\mathrm{R}=0$ is the common rotation axis passing through the centers of the rotating substrate heater and the rotating ablation target.

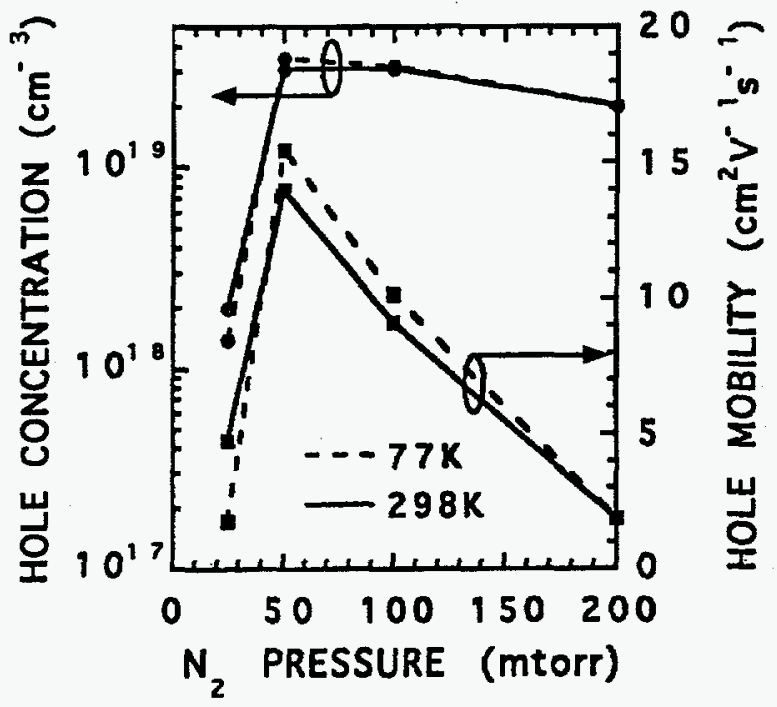

Figure 3. Hole concentration and mobility vs $\mathrm{N}_{2}$ pressure (at $1 \mathrm{sccm}$ flow) for p-ZnTe:N films grown at $\mathrm{T}=320^{\circ} \mathrm{C}$ and $\mathrm{E}_{\mathrm{d}}=0.66$ $\mathrm{J} / \mathrm{cm}^{2}$.

highest hole concentration $\left(\sim 7 \times 10^{19} \mathrm{~cm}^{-3}\right)$ and high hole mobility $\left(\sim 25-30 \mathrm{~cm}^{2} / \mathrm{V}-\mathrm{s}\right)$ in films $\sim 1$ $\mu \mathrm{m}$ thick. ${ }^{13}$ The combination of the RBS data of Fig. 1 with the Hall measurements discussed below suggests that, under appropriately controlled conditions, films with $\mathrm{Zn}: \mathrm{Te}$ stoichiometry very close to 1:1 can be grown by PLA of a stoichiometric ZnTe target.

Extensive RBS measurements also were carried out for PLA ZnSe films deposited by ablation of a stoichiometric $\mathrm{ZnSe}$ target onto $\mathrm{Si}(001)$ substrates at $\mathrm{T}=300^{\circ} \mathrm{C}$. The measured range of $\mathrm{Zn} / \mathrm{Se}$ ratios was very similar to that in Fig. 1, with the precise values apparently sensitive to both the ambient gas pressure and laser $E_{d}$. RBS results for $\mathrm{ZnSe}$ will be reported after an absolute calibration of the $\mathrm{Zn} / \mathrm{Se}$ ratio has been performed. ${ }^{18}$ Ablation of stoichiometric $\mathrm{ZnSe}$ through molecular $\mathrm{N}_{2}$ has not yet produced highly doped $\mathrm{p}-\mathrm{ZnSe}$, although the absence of sample charging during Auger electron spectroscopy measurements indicates that the PLA ZnSe films are doped to a low carrier concentration, but not high enough for good ohmic contact formation. ${ }^{18} \mathrm{ZnSe}$ is known to be much more sensitive to the formation of vacancies, antisite defects, and/or third phases (solubility limits) that make it much more susceptible to compensation of holes than is ZnTe. ${ }^{12-14}$

Doping from the Gas Phase: p-type ZnTe:N

The PLA film growth system, GaAs(001) substrate preparation, and film growth conditions used in these experiments have been described elsewhere recently.7,8,19 Briefly, both insulating and highly hole-doped p-ZnTe films were grown at $320^{\circ} \mathrm{C}$ by ablating a stoichiometric ZnTe target 
through $\mathrm{Ar}, \mathrm{N}_{2}$ and $\mathrm{N}_{2} /$ Ar gas mixtures, at a target-substrate separation, $\mathrm{D}_{\mathrm{ts}}$, of $10 \mathrm{~cm}$.

Hall effect measurements were used to determine the free hole concentration, $p$, and hole mobility, $\mu_{\mathrm{p}}$, at room temperature and at $77 \mathrm{~K}$. As shown in Fig. 3, the hole concentrations were nearly independent of temperature, indicating heavy doping and formation of an impurity conduction band. ZnTe films grown in pure Ar gas were electrically insulating, while ZnTe films grown in $\mathrm{N}_{2}$ /Ar mixtures had lower hole concentrations than shown in Fig. 3, consistent with doping that is controlled by the presence of $\mathrm{N}_{2}$. By slightly varying the growth conditions a maximum hole concentration $>1.1 \times 10^{20} \mathrm{~cm}^{-3}$ was achieved, approximately equaling the highest hole doping obtained in a wide band gap II-VI film by any growth method. 7,8

Figure 3 shows that the hole mobility is sharply peaked near a $\mathrm{N}_{2}$ pressure of 50 mtorr, with the hole concentration less sharply peaked. As shown in the next section, the peak in hole mobility is believed to be due primarily to lattice damage caused by the ablation beam's kinetic energy, at low $\mathrm{N}_{2}$ pressures, and to changes in the dominant deposition species and film-growth mode with increasing $\mathrm{N}_{2}$ pressures.

\section{Effect of Ambient Gas Pressure: Changes in the Dominant Film-Growth Species}

A combination of in situ RHEED and time-resolved ion probe observations have been used together with ex situ TEM images to determine why there is such a pronounced peak in the hole mobility (Fig. 3) as a function of $\mathrm{N}_{2}$ pressure. ${ }^{19}$

Figure 4 shows results of time-resolved ion current measurements at a distance of $10 \mathrm{~cm}$ from a $\mathrm{ZnTe}$ target, during ablation into $\mathrm{N}_{2}$ gas under film-growth conditions. 19 Geohegan et al. previously have used such measurements to show that for ablation of various materials into an ambient gas, the ablation plume quite generally splits into two or more components traveling at different average velocities and kinetic energies. ${ }^{20}$ They interpret the fastest pulse as ions that have undergone no collisions with gas molecules before arriving at the substrate. Although this fast pulse is exponentially attenuated by increasing the $\mathrm{N}_{2}$ gas pressure or the target-substrate separation, Fig. 4 shows that it remains significant for $\mathrm{ZnTe}$ ablation into $\mathrm{N}_{2}$ at $\mathrm{D}_{\mathrm{ts}}=10 \mathrm{~cm}$ until the $\mathrm{N}_{2}$ pressure is increased to $\sim 50$ mtorr, where the peak in hole mobility also occurs (Fig. 3 ).

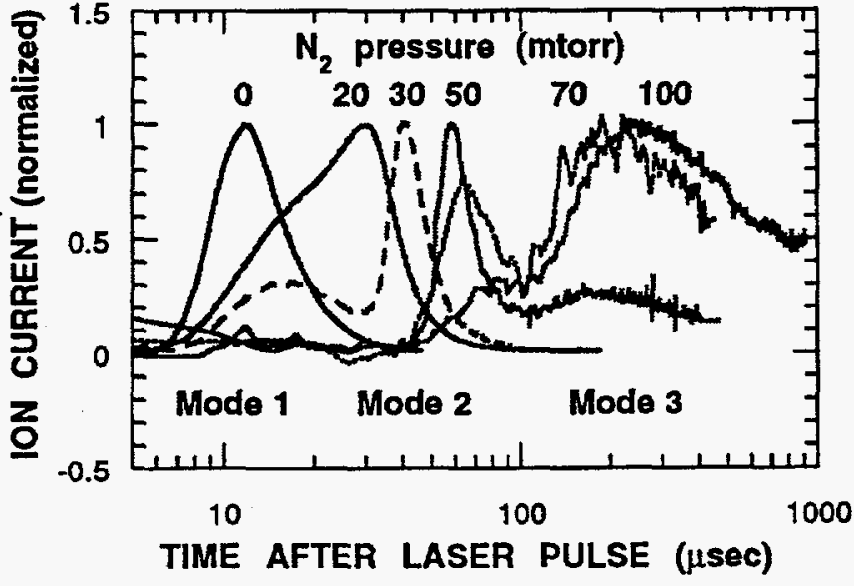

Figure 4. Ion currents measured at $\mathrm{D}_{\mathrm{ts}}=10 \mathrm{~cm}$ following ablation of $\mathrm{ZnTe}$ into $\mathrm{N}_{2}$ at various pressures.
This striking correlation suggests that the fast (vacuum) pulse of unscattered ions and atoms has sufficient kinetic energy to produce lattice displacement damage and point defects that reduce the hole mobility. ${ }^{7}$

In another paper of this symposium $^{19}$ Rouleau et al. have quantified this idea by deconvoluting the ion probe data of Fig. 4 into its constituent ion distributions, or dominant "modes" of incident species. At each $\mathrm{N}_{2}$ pressure either two or three exponentially-modified Gaussian distributions, each with a characteristic velocity and amplitude, were required to fit the ion probe data. For mode 1 (the

vacuum or collision-free ion pulse in Fig. 4) they found that $50 \%$ of the species have average velocities $\geq 0.75 \times 10^{6} \mathrm{~cm} / \mathrm{s}$, corresponding to kinetic energies $\geq 19 \mathrm{eV}(\geq 37 \mathrm{eV}$ ) for $\mathrm{Zn}$ (Te) atoms, more than enough to cause lattice damage. (Absorption and emission spectroscopy measurements also revealed that mode 1 is nearly all atomic species.) The most important conclusion of Rouleau et al. is that there are transitions as a function of $\mathrm{N}_{2}$ pressure from mode 1 dominance to mode 2 , at $\sim 15$ mtorr, and from mode 2 dominance to mode 3 , at $\sim 70$ mtorr. 19 The hole mobility and hole concentration maxima of Fig. 3 both lie in the region of mode 2 dominance. 
Mode 2 corresponds to $\mathrm{Zn}$ and Te kinetic energies of only a few eV or less, demonstrating that $\mathrm{p}-\mathrm{ZnTe}$ films of the highest quality are grown from an incident flux having relatively low kinetic energy.

However, Fig. 3 shows that increasing the $\mathrm{N}_{2}$ pressure beyond the region of mode 2 dominance degrades electrical properties, while Fig. 4 shows a third peak developing and dominating the ion current distribution for $\mathrm{N}_{2}$ pressures $\geq 70$ mtorr.

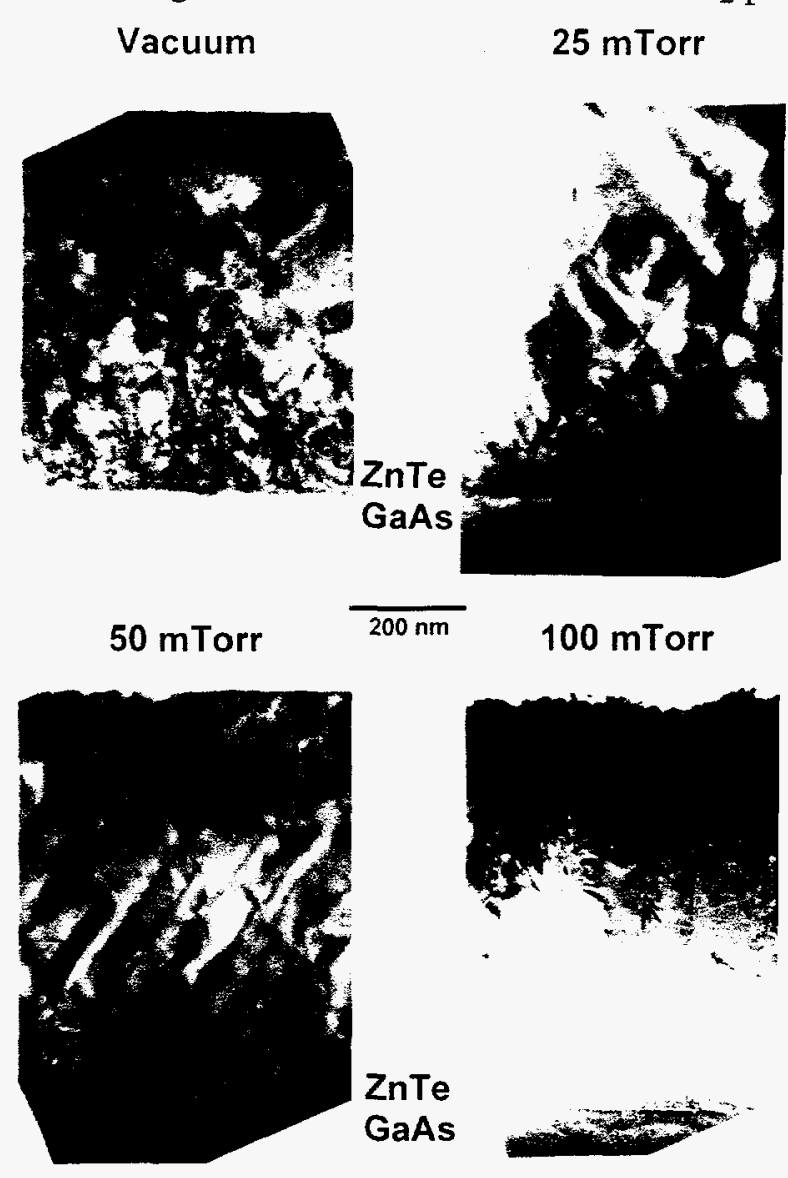

Figure 5. Cross-section TEM images of $\mathrm{ZnTe}$ films grown on $\mathrm{GaAs}(001)$ at $320^{\circ} \mathrm{C}$ under $\mathrm{N}_{2}$ pressures of $0,25,50$, and 100 mtorr (see text).

Figure 5 shows cross-sectional TEM images of p-ZnTe: $\mathrm{N}$ films grown at $\mathrm{N}_{2}$ pressures of $0,25,50$, and 100 mtorr. These images reveal that the primary cause for the degraded hole mobility near 100 mtorr is the transition to mode 3 growth, and that this transition probably marks the onset of significant cluster deposition. Ignoring the heavily dislocated region within $150 \mathrm{~nm}$ of the $\mathrm{ZnTe} / \mathrm{GaAs}$ heterointerface, which results from a nearly $8 \%$ lattice mismatch, microtwins (identified by selected area diffraction patterns) were found to be the dominant extended defects in the samples of Fig. 5. Rouleau et al. obtained microtwin areal densities for each of the samples shown in Fig. 5 by counting the total area of microtwins in the volume of $\mathrm{ZnTe} \geq 150 \mathrm{~nm}$ from the interface. 19 The densities were 8.3 $( \pm 1) \times 10^{4}, 11.3( \pm 2) \times 10^{4}$, and 15 $( \pm 2.5) \times 10^{4} \mathrm{~cm}^{2} / \mathrm{cm}^{3}$, for $\mathrm{N}_{2}$ pressures of 0,25 , and 50 mtorr. This result demonstrates an increasing density of extended defects with increasing $\mathrm{N}_{2}$ pressure in the low-pressure growth regimes dominated by modes 1 and 2 .

However, growth at an $\mathrm{N}_{2}$ pressure of 100 mtorr produced a distinctive change in film microstructure, to a columnar grain structure with grain diameters of -95 to $140 \mathrm{~nm}$ (see Fig. 5). Moreover, both the film surface and near-surface intergranular regions were decorated with spherical particles having diameters of $\sim 9.5$ to $17 \mathrm{~nm}$. TEM images of films grown at pressures between 50 and 100 mtorr have not yet been obtained. However, the ion probe data (Fig. 3) provide compelling evidence that the transition to mode 3 dominance occurs near 70 mtorr. The very low velocities of the species in the mode 3 ion pulse (Fig. 3), together with TEM images revealing a transition to columnar growth with spherical particles decorating films grown at 100 mtorr, all strongly suggest that films grown under conditions of mode 3 dominance are largely the product of cluster deposition.

\section{High Resolution X-ray Diffraction Studies: Crystallinity and Microstructure of p-ZnTe:N}

High resolution $x$-ray diffraction (HRXRD) measurements have been performed on ZnTe films grown in Ar and $\mathrm{N}_{2}$ at various pressures and at both high $\left(2.7 \mathrm{~J} / \mathrm{cm}^{2}\right)$ and low $\left(0.66 \mathrm{~J} / \mathrm{cm}^{2}\right)$ laser $\mathrm{E}_{\mathrm{d}}$ values. ${ }^{7} \mathrm{~A}$ combination of $\theta-2 \theta$ plots and $\phi$-scans of the $\mathrm{ZnTe}(404)$ reflection (to assess inplane epitaxy) revealed that all of the films were exclusively or predominantly in a single orientation, being fully epitaxial in three dimensions in the "cube on cube" alignment, with the film and substrate $<00 \ell>$ axes precisely aligned. Even for films grown at high $\mathrm{E}_{\mathrm{d}}$, only very small 
amounts $(<1 \%)$ of the $\mathrm{ZnTe}(311)$ and (111) orientations were present.

However, rocking curve $(\theta$-scan) measurements through the $\mathrm{ZnTe}(004)$ reflection revealed a significant mosaic spread $\Delta \theta \sim 0.5$ deg for a film grown in 50 mtorr Ar and $\Delta \theta \sim 1.1$ deg for films grown in 100 mtorr $\mathrm{N}_{2} .{ }^{7}$ Plots of the FWHM peak width $\Delta \mathrm{Q}$ vs. $\mathrm{Q}$ for the $(00 \mathrm{R})$ reflections had a steep slope for films grown in $100 \mathrm{mtorr}_{2}$, and a shallower slope for 50 mtorr Ar, revealing that most of the rocking curve width is due to strain. These results now can be understood from the new TEM measurements reported in the previous section. The greater rocking curve width for $\mathrm{ZnTe}: \mathrm{N}$ grown at $100 \mathrm{mtorr}$ is due to the columnar microstructure (Fig. 5) and to heavy $\mathrm{N}$ doping (see below). The smaller rocking curve width for films grown in 50 mtorr Ar apparently results from the smaller misalignments associated with the twin defect density.

The $\mathrm{p}-\mathrm{ZnTe}: \mathrm{N}$ film with the highest hole concentration and mobility had surface-normal and inplane lattice parameters of $6.0755( \pm 0.002) \AA$ and $6.078 \AA$, respectively, nearly identical but both $\sim 0.4 \%$ smaller than the bulk ZnTe value of $6.100 \AA$. A reduced in-plane lattice parameter also was found for another film grown in $\mathrm{N}_{2}$, but not for a film grown in Ar. These results strongly suggest that substantial nitrogen incorporation on substitutional sites is responsible for the lattice contraction in PLA ZnTe:N films, consistent with their highly p-type conductivity.

We note that there may also be two secondary causes for the decrease in hole mobility at high $\mathrm{N}_{2}$ pressures (Fig. 3). First, the kinetic and internal energies of incident species probably assist processes of surface diffusion, substitutional incorporation, and/or activation of $\mathrm{N}$ atoms on $\mathrm{Te}$ sites at intermediate $\mathrm{N}_{2}$ pressures, but these energies are reduced by gas-phase collisions at higher pressures. Second, the hole mobility may be decreased by the lattice distortion that accompanies the very high $\mathrm{N}$ incorporation in the solid phase at the higher $\mathrm{N}_{2}$ pressures.

\section{Mechanism for Nitrogen Doping During Pulsed Laser Ablation}

The interaction of the ablation plasma plume with molecular $\mathrm{N}_{2}$ was studied spectroscopically in the same chamber used for ion current measurements. ${ }^{21}$ Optical emission was monitored using a $1.33-\mathrm{m}$ monochromator equipped with an 1800 groove $/ \mathrm{mm}$ holographic grating, a gated intensified diode array, and a photomultiplier tube. The principal result of spectroscopic measurements at a distance of $7 \mathrm{~cm}$ from the ZnTe target during PLA in molecular $\mathrm{N}_{2}$ was that no emission was observed from either excited atomic nitrogen or excited molecular nitrogen. On the other hand, when a nitrogen glow discharge was established between parallel $\mathrm{Cu}$ plates in the same chamber, strong near-infrared $\mathrm{N}^{+}$atomic emission lines and an $\mathrm{N}_{2}{ }^{+}$emission band were seen. ${ }^{7}$

The lack of atomic $\mathrm{N}$ emission from the PLA plasma in our experiments contrasts with the recent observation by Vaudo et al. ${ }^{22}$ of strong near-IR atomic $\mathrm{N}$ lines emitted from the nitrogen plasma produced by an RF source that is used for N-doping during MBE growth. They concluded that atomic $\mathrm{N}$ (not molecular $\mathrm{N}_{2}$ ) is responsible for RF plasma p-type doping of $\mathrm{ZnSe}$ (and $\mathrm{ZnTe}$ ) under MBE growth conditions. ${ }^{22}$ Our experiments do not directly rule out the presence of atomic $\mathrm{N}$ in the ground state during PLA, but this seems unlikely. The dissociation energy of $\mathrm{N}_{2}$ is nearly $10 \mathrm{eV}$; if PLA produced atomic $\mathrm{N}$ by dissociation of $\mathrm{N}_{2}$, then we also would expect to see emission from excited $\mathrm{N}_{2}$, which we did not. A simple explanation for the absence of atomic $\mathrm{N}$ in PLA experiments is that Vaudo et al. also found that the relative intensities of the near-IR atomic $\mathrm{N}$ and molecular $\mathrm{N}_{2}$ emissions depended sensitively on MBE chamber pressure, and the atomic $\mathrm{N}$ emission nearly disappeared when the pressure was raised from $2 \times 10^{-6}$ torr to $5 \times 10^{-5}$ torr. ${ }^{22}$ Consequently, even if atomic $\mathrm{N}$ were produced in the ablation plume, it would be short-lived at the 25-100 mtorr pressures used here, due to the increased probability of collisions and rapid recombination, and so is unlikely to be involved in doping at the substrate.

The most likely nitrogen-doping mechanism during PLA growth is the direct kinetic energyenhanced reaction of $\mathrm{Zn}$ atoms with $\mathrm{N}_{2}$ to form $\mathrm{Zn}-\mathrm{N}$ compounds, either in the incident ablation flux or with $\mathrm{N}_{2}$ molecules that are adsorbed on the growing film surface. Experiments to distinguish between these two possibilities are planned: We note that Nakao and Uenoyama have suggested 23,24 that $\mathrm{N}_{2}$ may be able to adsorb on the growing film surface in the metastable $\mathrm{A}^{3} \sum_{\mathrm{u}}{ }^{+}$ state and subsequently dissociate. Vaudo et al. ruled out this pathway as unlikely in the presence of a thermal MBE-growth atomic flux. ${ }^{22}$ However, the kinetic energies of ablated atoms and ions 
are sufficient, over a range of $\mathrm{N}_{2}$ pressures, to supply the $3.9 \mathrm{eV}$ needed to dissociate the $\mathrm{A}^{3} \sum_{\mathrm{u}}{ }^{+}$ state, and so this mechanism needs to be considered for PLA growth.

\section{Doping from Atoms Incorporated in the Ablation Target}

Shen and Kwok have reported 10 both $\mathrm{n}$ - and p-type doping of (001)-oriented cubic (zinc blende) $\mathrm{CdS}$ films grown by PLD at $300-400^{\circ} \mathrm{C}$ on semi-insulating (001) GaAs substrates. Films were grown in both 8 mtorr and 100 mtorr Ar, using target-substrate separations, $D_{\mathrm{ts}}$, of $10 \mathrm{~cm}$ and $5 \mathrm{~cm}$, to produce film deposition rates of $0.05 \AA /$ pulse and $0.4 \AA /$ pulse, respectively. $\mathrm{Li}_{3} \mathrm{~N}$ or In powders were added to pressed-powder CdS targets for p-type and n-type doping, respectively.

A Li concentration of 1 mole \% in the target produced a p-type CdS film. However, because film resistivities generally were high, Hall effect measurements were possible only for higher dopant concentrations. A target with 11 mole \% Li produced the lowest resistivity CdS:Li film, with a hole concentration $\mathrm{p} \sim 1.1 \times 10^{17} \mathrm{~cm}^{-3}$ and mobility $\mu_{\mathrm{p}} \sim 9.8 \mathrm{~cm}^{2} / \mathrm{V}$-s. For n-type doping, the lowest resistivity CdS:In film was obtained using a target that contained 9 mole \% In and produced a high electron concentration in the mid- $10^{20} \mathrm{~cm}^{-3}$ range. The highest Hall mobilities obtained for $n-$ and p-CdS films were 230 and $22 \mathrm{~cm}^{2} / \mathrm{V}-\mathrm{s}$, about $2 / 3$ and $1 / 2$, respectively, of the values expected for single crystal CdS. ${ }^{10}$

Shen and Kwok also reported p-type doping of $\mathrm{ZnS}, \mathrm{ZnSe}$, and CdSe using Li-doped targets. ${ }^{9}$ These films apparently were grown using $D_{t s}=5 \mathrm{~cm}$, in 100 mtorr Ar, at $E_{d}=1.5 \mathrm{~J} / \mathrm{cm}^{2} .10$ Thermopower (hotprobe) measurements revealed conversion from high resistivity n-type behavior to p-type conduction for Li concentrations as low as 0.5 mole \%. SEM studies showed that the films' surface morphology degraded for Li concentrations in the target greater than 6 mole \% for $\mathrm{ZnS}$ and $\mathrm{ZnSe}$, or greater than 3 mole \% in the target for CdS and CdSe. The lowest resistivities obtained were $\sim 102 \Omega$-cm for $\mathrm{ZnS}: \mathrm{Li}(9 \mathrm{~mole} \% \mathrm{Li}$ in the target) and $\sim 5.8 \Omega-\mathrm{cm}$ for $\mathrm{CdS}: \mathrm{Li}$ (11 mole \% Li). ${ }^{9}$ Hall effect measurements were not made for $\mathrm{ZnS}$ and $\mathrm{ZnSe}$ because of difficulties in making ohmic contacts. ${ }^{9}$

The concentrations of In and $\mathrm{Li}$ atoms in the targets used by Shen and Kwok were much higher than the carrier concentrations in their films. Relatively smooth film morphologies were obtained for deposition from a CdS target containing 9 mole \% In, and from a $\mathrm{ZnS}$ target containing 6 mole $\% \mathrm{Li}$, suggesting that actual dopant atom concentrations (dopant incorporation) in the films were much lower than in the targets. The carrier concentrations achieved using $\mathrm{Li}$ also suggest that PLA at least partially overcomes (though it may not prevent) the formation of $\mathrm{Li}$ interstitials that act as compensating donors. ${ }^{9}$ The electrical measurements carried out to date imply that either (a) low melting point dopants such as In $\left(156.6^{\circ} \mathrm{C}\right)$ and $\mathrm{Li}\left(180.6^{\circ} \mathrm{C}\right)$ have very low sticking coefficients 9 (contrary to the stoichiometric deposition normally expected of pulsed laser deposition) and so are not incorporated in films, or (b) these dopant atoms are incorporated but are electrically inactive. Direct measurements of both dopant incorporation and carrier concentration are needed to resolve this question for In and $\mathrm{Li}$.

\section{EPITAXIAL GROWTH OF n-TYPE CuInSe 2}

Highly oriented, n-type epitaxial $\mathrm{CuInSe}_{2}$ (CIS) films were grown on semi-insulating $\mathrm{GaAs}(\mathrm{Cr})$ substrates whose surface normal was tilted $2^{\circ}$ from [001] toward [110]. The growth chamber was the same as, and the growth conditions and techniques were similar to, those used to grow p-ZnTe:N. A 1-inch diam CIS target was prepared by cold-pressing $99.999 \%$ purity CIS powder at $\sim 60,000$ psi. The $\mathrm{KrF}$ laser was operated at $8 \mathrm{~Hz}$ and focused to give $E_{d}=0.66 \mathrm{~J} / \mathrm{cm}^{2}$ on the target. Substrates were indium-bonded to the rotating heater face and located $10 \mathrm{~cm}$ from the target. Films were grown in a background pressure of $40 \mathrm{mtorr} \mathrm{Ar}$ gas in order to reduce the ablation beam's kinetic energy into the range for which the best electrical properties were obtained for p-ZnTe:N (see Fig. 3). RHEED observations during and after ZnTe growth also had revealed that a well-developed, "streaky" (2D) RHEED pattern could be obtained for Ar pressures up to 40 mtorr, but the RHEED pattern degenerated into more complex spotty patterns at higher pressures. ${ }^{19}$ 

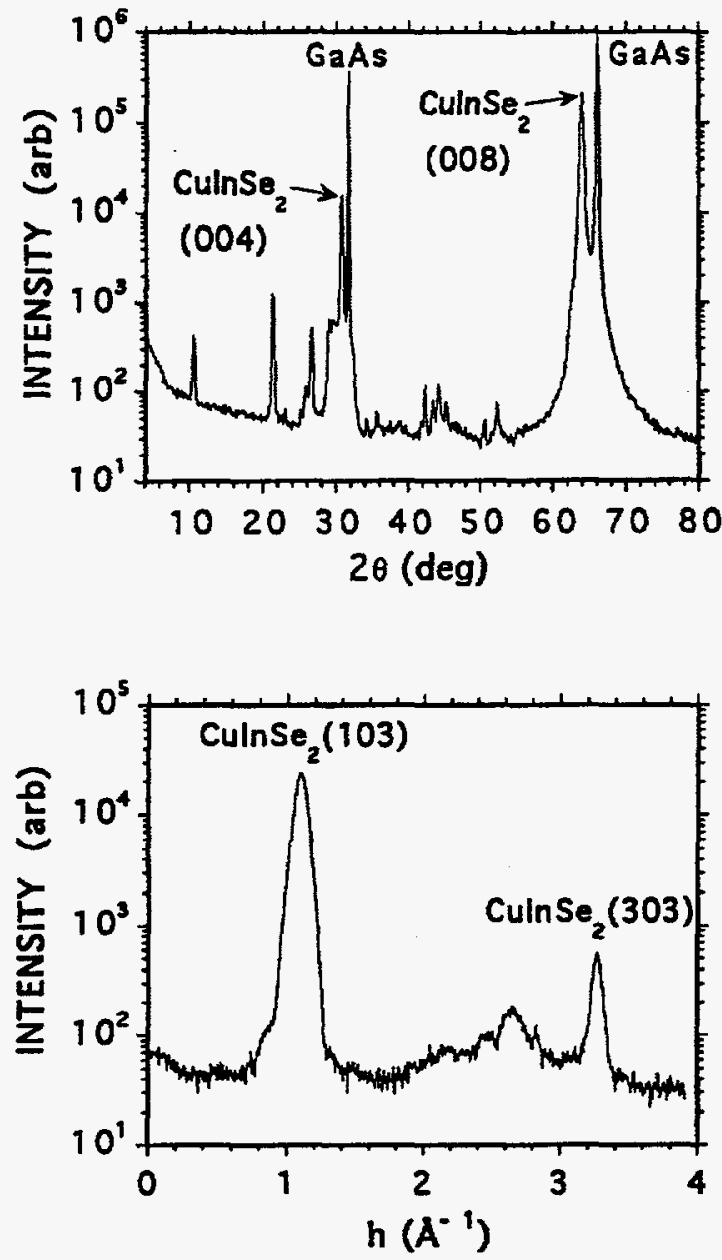

Figure 6. (Top) Diffracted x-ray intensity vs $2 \theta$ for epitaxial n-CuInSe 2 . (Bottom) h-scan at $\ell=3 \mathrm{c}^{*}$.
Figure 6 shows high-resolution $\mathrm{x}$-ray diffraction results for the best CIS film, grown at $\mathrm{T}=350^{\circ} \mathrm{C}$. The film and substrate $(00 \mathrm{l})$ axes are aligned. Strong diffraction peaks of the pure chalcopyrite phase were observed, with no peaks indicating a more complex ordering (e.g., of vacancies). The measured lattice constants (a, b = 5.776 $\AA, c=11.636 \AA$ ) are close to those of bulk CIS (a, b = 5.782 $\AA, c=11.619$ $\AA$, JCPDS 40-1487), with the in-plane CIS lattice constants slightly contracted and the c-axis slightly expanded, as expected for epitaxial growth on GaAs $(\mathrm{a}=5.653 \AA$ ). Several scans also were made to assess the extent of in-plane alignment between film and substrate, with the result that the CIS film is fully aligned epitaxially in the "cube on cube" orientation (see Fig. 6, bottom).

Fig. 6(top) also shows a number of relatively weak diffraction peaks that arise from a high areal density of particulates (observed by SEM) on this film. Six of the eight weak peaks in Fig. 6 (top) were indexed to InSe (JCPDS 12-118). The particulates resulted from the use of a cold-pressed powder target in these initial CIS growth experiments.

Hall effect measurements revealed that the CIS film of Fig. 6 was doped n-type, with $\mathrm{n}=6 \times 10^{17} \mathrm{~cm}^{-3}$ and $\mu_{\mathrm{n}}=55 \mathrm{~cm}^{2} / \mathrm{V}$-s. The source of n-type doping is unknown at this time.

These initial results are significant because the $350^{\circ} \mathrm{C}$ growth temperature for a fully epitaxial and n-type CIS film is $\geq 175^{\circ} \mathrm{C}$ lower than (i) the temperatures at which polycrystalline CIS is grown for photovoltaic cells (by a

coevaporation and partial melting process, to produce columnar grains) ${ }^{15,16}$ as well as (ii) the 520 $580^{\circ} \mathrm{C}$ temperature range used for recent PLA growth of $\mathrm{p}$ - and n-type polycrystalline CIS films. ${ }^{25}$ Experiments currently are being prepared to determine if it is possible to use PLA to grow a broad range of epitaxial CIS films at low temperatures and to dope them p- and n-type for studies of intrinsic materials properties and doping mechanisms.

\section{DOPED EPITAXIAL GaN AND InN FILMS}

Feiler, Williams, and collaborators recently demonstrated PLA growth and doping of column-III nitride epitaxial films. ${ }^{4,5} \mathrm{AlN}, \mathrm{GaN}$, and InN were grown epitaxially on sapphire $(0001)$ substrates at temperatures of $460^{\circ} \mathrm{C}-520^{\circ} \mathrm{C}$, much lower than the temperatures reported for most of the epitaxial nitride films grown by MOCVD or MBE. The most intriguing discovery by Feiler et al. was that their GaN films were doped either n-type or p-type, depending on whether the ambient atmosphere was high vacuum or $\sim 10$ mtorr nitrogen $\left(\mathrm{N}_{2}\right)$, respectively. 4,5 Electrically conducting p-type InN films also were grown, while the AlN films were insulating. ${ }^{4}$

Nitride films $\sim 90-400 \mathrm{~nm}$ thick were deposited by $1 . \mathrm{Hz} \mathrm{KrF}$ laser irradiation of targets that had been prepared by cold-pressing column-III nitride powders. The focused laser energy density was $1.5-3.5 \mathrm{~J} / \mathrm{cm}^{2}$ and the target-substrate separation was only $3 \mathrm{~cm}$ so that the substrate and growing film were bathed in the energetic fluorescent part of the ablation plasma plume. 26

AIN and GaN films were single crystalline in the same orientation, with the film (0001) II sapphire(0001) and the film [1100] II sapphire[1120].4 Thus, the AIN and GaN films were rotated 
by $90^{\circ}$ in-plane with respect to the sapphire substrate. This orientation places the films in compression in the in-plane direction and minimizes their lattice mismatches to sapphire(0001). The InN films also were highly oriented, with the film and substrate (0001) planes parallel. The dominant in-plane alignment was InN[1100] II sapphire[1010], i.e., the $\operatorname{InN}$ film was rotated $60^{\circ}$ with respect to the substrate. However, for InN the $\mathrm{x}$-ray diffraction $\phi$-scans contained broad peaks and high background scattering between peaks, showing that a large number of small crystallites with random in-plane orientations also were present. (These were presumably particulates from the cold-pressed powder target, seen by SEM.) The dominant $60^{\circ}$-rotated InN orientation also minimizes the lattice mismatch of InN to sapphire but places InN under tensile strain in the in-plane direction.

GaN films grown in high vacuum to thicknesses of $90 \mathrm{~nm}$ and $190 \mathrm{~nm}$ were n-type with an electron concentration and mobility of $1 \times 10^{18} \mathrm{~cm}^{-3}$ and $300 \mathrm{~cm}^{2} / \mathrm{V}-\mathrm{s}$, respectively. 4,5 In contrast, a GaN film grown in 10 mtorr of nitrogen $\left(\mathrm{N}_{2}\right)$ was p-type with a hole concentration and mobility of $7.5 \times 10^{18} \mathrm{~cm}^{-3}$ and $150 \mathrm{~cm}^{2} / \mathrm{V}$-s, respectively. In both cases Feiler et al. attribute the conductivity to unintentional impurity doping. Silicon, at $\sim 100 \mathrm{ppm}$, was the major impurity in the $\mathrm{GaN}$ powder used for target fabrication and is an n-type dopant in $\mathrm{GaN}$. It is thought that $\sim 1 \mathrm{ppm}$ carbon monoxide ( $\mathrm{CO}$ ) in the $\mathrm{N}_{2}$ ambient gas used during ablation may have resulted in carbon incorporation in the films grown in $\mathrm{N}_{2}$, and a net p-type doping that compensated the $n$-type doping by Si impurities.

Feiler et al. point out that for both n-type and p-type PLD GaN films, the carrier mobility values are considerably higher than expected from baseline data for $\mathrm{GaN}$ films grown earlier by other methods. 27,28 A 190 nm-thick GaN film grown by PLD in vacuum had an $x$-ray rocking curve width of 280 arc sec for the GaN(0002) peak, very good for a film of this thickness. Thus, the combination of electrical and $\mathrm{x}$-ray measurements demonstrates that at temperatures near $500^{\circ} \mathrm{C}$ PLD produces epitaxial GaN films with crystallinity and electrical properties that rival films grown by other methods at much higher temperatures, especially when the thinness of the PLD films and their lack of use of a buffer layer is taken into account. 4,5

The InN films prepared by Feiler et al. were strongly p-type both when grown in vacuum $\left(\mathrm{p}=6.5 \times 10^{20} \mathrm{~cm}^{-3}\right.$ and $\left.\mu_{\mathrm{p}}=30 \mathrm{~cm}^{2} / \mathrm{V}-\mathrm{s}\right)$ and in a $\mathrm{N}_{2}$ ambient $\left(\mathrm{p}=4.7 \times 10^{19} \mathrm{~cm}^{-3}\right.$ and $\left.\mu_{p}=240 \mathrm{~cm}^{2} / \mathrm{V}-\mathrm{s}\right)$. This contrasts with the n-type doping of InN that is obtained using conventional growth techniques, for which the electron doping is believed to be due to nitrogen vacancies. The source of hole doping in the PLD InN films is not known.

\section{SUMMARY AND CONCLUSIONS}

The series of recent experiments described above demonstrate that laser ablation is a promising technique for the growth of complex compound semiconductors. The use of high-density, hotpressed ablation targets reduces the areal density of particulates to a level that is negligible for exploratory materials research and may be acceptable for some device applications. RBS measurements, though limited by $\sim 1 \%$ precision show that stoichiometric epitaxial films can be grown by ablation of a stoichiometric compound semiconductor target, though congruent transfer of material does appear to be sensitive to ambient gas pressure and pulsed laser energy density.

Highly p-type ZnTe: $\mathrm{N}$ films have been grown by ablating a $\mathrm{ZnTe}$ target through molecular $\mathrm{N}_{2}$, approximately equaling the highest hole concentrations $\left(-10^{20} \mathrm{~cm}^{-3}\right)$ obtained by any method for a wide band gap II-VI semiconductor. These experiments demonstrate the general feasibility of doping compound semiconductors from the gas phase during PLA. (Earlier experiments showed that $\mathrm{ZnSe}_{1-\mathrm{x}} \mathrm{S}_{\mathrm{X}}$ film composition also could be controlled via the partial pressure of ambient $\mathrm{H}_{2} \mathrm{~S}$ gas. ${ }^{6}$ ) The heavy hole doping of $\mathrm{ZnTe}: \mathrm{N}$ is accompanied by a uniform lattice contraction, consistent with nitrogen incorporation on substitutional lattice sites and the resulting p-type conductivity. However, atomic nitrogen is not observed in the laser-generated plasma. The high nitrogen doping apparently occurs by a new mechanism, believed to be direct kinetic energyenhanced reactions of $\mathrm{Zn}$ with $\mathrm{N}_{2}$, either adsorbed on the growing surface or in the gas phase.

A combination of RHEED, ion probe, and TEM measurements have revealed a succession of transitions, controlled by $\mathrm{N}_{2}$ pressure, between distinctive growth regimes in which species with quite different characteristics dominate the incident ablation flux. ${ }^{19}$ Clearly 2D (streaky) RHEED patterns were obtained for $\mathrm{N}_{2}(\mathrm{Ar})$ pressures of $<30(<40)$ mtorr, with spotty (3D) patterns for 
pressures $\geq 50$ mtorr. ${ }^{19}$ Time-resolved ion current measurements have been deconvoluted to reveal three distinct peaks in the ion (and presumably the atomic) flux as a function of $\mathrm{N}_{2}$ pressure. The fast peak (mode 1), dominant for $\mathrm{N}_{2}$ pressures $\leq 15 \mathrm{mtorr}$, is due to unscattered ions having kinetic energies sufficient to do lattice displacement damage to the growing film. Mode 2, dominant for pressures up to $\sim 70$ mtorr, consists of ions and atoms with kinetic energies of only a few eV or less, which seem ideal for energetic beam-assisted film growth and near-surface chemical reactions. The peak in hole mobility for $\mathrm{p}-\mathrm{ZnTe}: \mathrm{N}$ films is reached near 50 mtorr $\mathrm{N}_{2}$ pressure, where mode 2 is dominant, though cross-section TEM images reveal an increasing density of extended defects (microtwins) from vacuum up to 50 mtorr. At higher $\mathrm{N}_{2}$ pressures, TEM, ion probe, and electrical properties measurements all reveal a transition to a new growth regime, which is believed to be the onset of significant cluster deposition, near 70 mtorr $\mathrm{N}_{2}$ pressure. Films exhibit a columnar microstructure and decreasing hole mobility in this regime. The broadening found in high resolution XRD rocking curves is consistent with the microstructural changes observed by TEM.

Both n- and p-type doping of II-VI films by incorporating column-I ( $\mathrm{Li}$ ) or column-III (In) dopant atoms directly in ablation targets also have been demonstrated by Shen and Kwok. 9,10 However, quite high $(\sim 10$ mole $\%) \mathrm{Li}$ and In concentrations were required in the targets to obtain films with sufficiently low resistivity to make ohmic contacts and carry out Hall effect measurements. Thermopower measurements suggest p-type doping of $\mathrm{ZnS}, \mathrm{ZnSe}$, and CdSe, but Hall measurements were not made because of difficulties in obtaining ohmic contacts. The fact that the dopant atom concentrations in ablation targets were so much larger than carrier concentrations in films suggests either that dopants were not incorporated efficiently in films or that they were electrically inactive. Direct measurements of dopant incorporation and carrier concentrations in films are needed, preferably with use of high-density targets, to resolve this question.

Doped and fully epitaxial films of $\mathrm{CuInSe}_{2}$ and column-III nitrides also have been grown. The growth of epitaxial $\mathrm{n}-\mathrm{CuInSe} 2$ at $350^{\circ} \mathrm{C}$ is promising because this temperature is $\sim 175^{\circ} \mathrm{C}$ below those at which polycrystalline $\mathrm{CuInSe}_{2}$ films have been grown previously for photovoltaic energy conversion. This work suggests that it may be possible to grow and dope a variety of epitaxial IIII-VI films at low temperatures and to study their intrinsic properties and doping mechanisms.

The $\sim 500^{\circ} \mathrm{C}$ temperature at which doped $\mathrm{GaN}$ and $\mathrm{InN}$ films were grown on sapphire $(0001)$ by PLA, without use of a buffer layer, is significantly lower than the temperatures at which most epitaxial nitride films have been grown by MOCVD or MBE. The PLA films were grown at a target-substrate separation of only $3 \mathrm{~cm}$ and so were immersed in the excited laser ablation plasma. These conditions produced GaN films whose crystallinity and electrical properties rival those of films grown at much higher temperatures by other methods. The implication is that PLD growth directly in the ablation plume, with both kinetic and internal internal energies available, must be an important factor permitting growth of high quality $\mathrm{GaN}$ at low temperatures. Indeed, direct comparisons of the microstructure of MOCVD and PLD GaN films, as a function of film thickness, support the idea that PLD and MOCVD growth mechanisms for GaN must differ. 4,5 The growth of strongly p-type InN films also suggests distinctly different growth conditions and doping behavior during PLD than with conventional growth methods. ${ }^{4}$ The occurrence of accidental doping by impurities, both in the ablation target and in the gas phase, in the GaN experiments promises considerable versatility and convenience in future use of PLA to grow and dope these materials.

Finally, the examples cited above of new and distinctly different growth and doping behavior under PLA conditions emphasizes the potential importance of a new growth environment and the continuing need for in situ diagnostic measurements in order to develop a fundamental understanding of how this environment influences compound semiconductor growth and doping.

\section{ACKNOWLEDGEMENT}

This research was sponsored by the Division of Materials Science, U. S. Department of Energy, under contract no. DE-AC05-84OR21400 with Lockheed Martin Energy Systems, Inc. and supported in part by an appointment to the Oak Ridge National Laboratory Postdoctoral Research Associates Program administered jointly by the Oak Ridge National Laboratory and the Oak Ridge Institute for Science and Education. 


\section{REFERENCES}

1. See Pulsed Laser Deposition of Thin Films, ed. by D. B. Chrisey and G. K. Hubler, John Wiley and Sons, New York, 1994.

2. For selected references to early PLD of semiconductors, see the bibliography by $\mathrm{K}$. L. Saenger, p. 581-604 in Ref. 1.

3. H. J. Möller, Chap. 8 in Semiconductors for Solar Cells, Artech House, Norwood, MA, 1993.

4. "Pulsed Laser Deposition of Epitaxial AIN, GaN, and InN Thin Films on Sapphire(0001)," D. Feiler, R. S. Williams, A. A. Talin, H. Yoon, and M. S. Goorsky, submitted to J. of Crystal Growth..

5. "Pulsed Laser Deposition of Epitaxial GaN on Sapphire(0001)," D. Feiler, R. S. Williams, A. A. Talin, H. Yoon, K. Matney, and M. S. Goorsky, submitted to Applied Physics Letters.

6. J. W. McCamy, D. H. Lowndes, and J. D. Budai, Appl. Phys. Lett. 63, 3008 (1993).

7. D. H. Lowndes, C. M. Rouleau, J. W. McCamy, J. D. Budai, D. B. Poker, D. B. Geohegan, A. A. Puretzky, and Shen Zhu, p. 85 in Film Synthesis and Growth Using Energetic Beams, ed. by H. A. Atwater, J. T. Dickinson, D. H. Lowndes, and A. Polman, Materials Research Society, Pittsburgh, PA, 1995.

8. C. M. Rouleau, D. H. Lowndes, J. W. McCamy, J. D. Budai, D. B. Poker, D. B. Geohegan, A. A. Puretzky, and Shen Zhu, Appl. Phys. Lett. 67, 2545 (1995).

9. W. P. Shen and H. S. Kwok, p. 91 in Film Synthesis and Growth Using Energetic Beams, ed. by H. A. Atwater, J. T. Dickinson, D. H. Lowndes, and A. Polman, Materials Research Society, Pittsburgh, PA, 1995.

10. W. P. Shen and H. S. Kwok, p. 173 in New Materials for Advanced Solid State Lasers, ed. by B. H. T. Chai, S. A. Payne, T. Y. Fan, and A. Cassanho, Materials Research Society, Pittsburgh, PA, 1994.

11. J. T. Cheung and J. Madden, J. Vac. Sci. Technol. B5, 705 (1987); see also J. T. Cheung, chap. 22 in Ref. 1.

12. R. M. Park et al., Appl. Phys. Lett. 57, 2127 (1990).

13. S. O. Ferreira et al., J. Cryst. Growth 140, 282 (1994).

14. T. Baron et al., Appl. Phys. Lett. 65, 1284 (1994).

15. J. R. Tuttle et al., Progress in Photovoltaics 3, 235 (1995); M. A. Contreras et al., Prog. in Photovoltaics 2, 287 (1994); A. M. Gabor et al., 12th NREL PV Program Review, AIP Conf. Proc. 303, 59 (1994).

16. H. W. Schock, Optoelectronics-Devices and Technologies 9, 511 (1994); H. W. Schock, Solar Energy Materials and Solar Cells 34, 19 (1994); T. Walter and H. W. Schock, 12th NREL PV Program Review, AIP Conf. Proc. 303, 67 (1994).

17. J. T. Cheung, Chap. 1 in Ref. 1.

18. D. H. Lowndes, C. M. Rouleau, J.-W. Park et al., manuscript in preparation.

19. See the paper by C. M. Rouleau, D. H. Lowndes, M. A. Strauss, S. Cao, A. J. Pedraza, D. B. Geohegan, A. A. Puretzky, and F. Allard, this symposium proceedings.

20. D. B. Geohegan and A. A. Puretzky, p. 21 in Film Synthesis and Growth Using Energetic Beams, ed. by H. A. Atwater, J. T. Dickinson, D. H. Lowndes, and A. Polman, Materials Research Society, Pittsburgh, PA, 1995.

21. D. B. Geohegan, p. 115 in Pulsed Laser Deposition of Thin Films, ed. by D. B. Chrisey and G. K. Hubler, Wiley, New York, 1994.

22. R. P. Vaudo, J. W. Cook, Jr., and J. F. Schetzina, J. Cryst. Growth 138, 430 (1994).

23. T. Nakao and T. Uenoyama, Jpn. J. Appl. Phys. 32, 660 (1993).

24. T. Uenoyama, T. Nakao, and M. Suzuki, J. Cryst. Growth 138, 301 (1994).

25. R. Schaffler, M. Klose, M. Brieger, H. Dittrich, and H. W. Schock, Materials Science Forum 173-174, 135 (1995).

26. D. Feiler, personal communication.

27. T. D. Moustakas and R. J. Molnar, Mat. Res. Soc. Symp. Proc. 281, 753 (1993).

28. S. Nakamura, M. Takashi, M. Senoh, and N. Iwasa, Jpn. J. Appl. Phys. 31, L139 (1992). 